\title{
The Effect of IntelliDrive on the Efficiency of Highway Transportation Systems
}

\author{
Mohammad Nekoui, ${ }^{1}$ Hossein Pishro-Nik, ${ }^{1}$ and Daiheng Ni² \\ ${ }^{1}$ Department of Electrical and Computer Engineering, University of Massachusetts, Amherst MA 01003, USA \\ ${ }^{2}$ Department of Civil and Environmental Engineering, University of Massachusetts, Amherst MA 01003, USA
}

Correspondence should be addressed to Mohammad Nekoui, nekoui@ecs.umass.edu

Received 15 August 2010; Accepted 6 December 2010

Academic Editor: Zainab Zaidi

Copyright (c) 2011 Mohammad Nekoui et al. This is an open access article distributed under the Creative Commons Attribution License, which permits unrestricted use, distribution, and reproduction in any medium, provided the original work is properly cited.

\begin{abstract}
Recently, the IntelliDrive initiative has been proposed by the US Department of Transportation (USDOT) to enhance on-road safety and efficiency. In this study, we provide a mathematical framework which predicts the effect of such technologies on the efficiency of multilane highway systems prior to their real-life deployment. Our study shall encompass mixed traffic conditions in which a variety of assisted, automated and unequipped vehicles coexist. We show that intervehicular communications improves the flow of vehicles by reducing the perception-reaction (P-R) times of drivers and, in some cases, allowing for more efficient lane-changing operations. As we shall see, unlike the latter, the former effect of IntelliDrive on driver P-R time is always there, regardless of the specific traffic conditions.
\end{abstract}

\section{Introduction}

With the ever increasing production of vehicles and their inevitable role in everyday life, transportation systems are drawing the attention of industry and academia more than any other time. Despite the undeniable beneficial aspects of transportation systems, there are numerous factors by which they impair our everyday life. Without any doubt, many of us have experienced being trapped in heavy traffic, wasting our time and energy resources. Traffic congestion wastes 40 percent of travel time on average, unnecessarily consumes about 2.3 billion gallons of fuel per year, and adversely impact the environment [1]. More importantly, traffic accidents are held responsible for a good portion of death causes. Annually, more than 40,000 people are killed and much more injured in highway traffic accidents in the United States alone [2]. IntelliDrive [3] - formerly known as Vehicle Infrastructure Integration (VII)—a major initiative at the United States Department of Transportation (USDOT), proposes to use Dedicated Short Range Communications (DSRCs) to establish vehicle-vehicle and vehicleroadside communications to deliver timely information to save lives, reduce congestion, and improve quality of life. The network of communicating vehicles forms a Vehicular AdHoc Network (VANET) on roads. VANET is an emerging area, and due to the potentially dramatic improvements it renders in terms of safety, highway efficiency, and driver convenience, has attracted attention from both academia and industry in the US, EU, and Japan. The most important feature of VANETs is their ability to extend the horizon of drivers and on-board devices and thus to improve road traffic safety, efficiency, and comfort. VANET will enable a wide range of novel applications such as accident avoidance messaging, congestion sensing, ramp metering, and general information services [4-6]. The allocation of $75 \mathrm{MHz}$ in the $5.9 \mathrm{GHz}$ band for DSRC may also enable future delivery of rich media content to vehicles at short to medium ranges via both intervehicle and roadside-vehicle communications.

In the near future, a traffic stream may consist of mixed vehicles operated under different driving modes: a vehicle may be operated without IntelliDrive assistance "unequipped", by a human driver with IntelliDrive assistance "assisted" or by an IntelliDrive-enabled automated system which is itself in charge of driving "automated". The 
IntelliDrive Initiative envisions that each future vehicle will be equipped with an On-Board Equipment (OBE) which includes a DSRC transceiver, a Global Positioning System (GPS) receiver, a processing unit, and possibly appropriate sensing accessories. Also equipped with similar devices, RoadSide Equipment (RSE) will be deployed at selected roadside locations. Therefore, vehicles will be able to communicate with each other and with the roadside by means of DSRC. As a result, assisted drivers will be able to respond to their driving environment earlier, that is, shorter PerceptionReaction (P-R) times than drivers without IntelliDrive assistance. Here, relevant information of a vehicle such as its location, speed, and acceleration are transmitted via its DSRC radio to its surrounding vehicles. The payload size of such a status packet is in the order of a (few) hundred bytes for ordinary safety applications [5]. This packet is sent with a frequency equivalent to the GPS update rate $(1-10 \mathrm{~Hz})$. However, the packet throughput relies on the total number of nodes and the physical characteristics of the wireless channel. With this setting, a vehicle that has a sufficient amount of information regarding its surroundings is typically informed about a specific traffic condition much sooner. Hence, it takes it less time to perceive its leader vehicle's sudden braking when that happens. Also, as the attention of assisted drivers has already been drawn to the condition before the driver is needed to react, the variance in his or her perception time (and hence the variance of P$\mathrm{R}$ time) is much lower than drivers of unequipped vehicles. The above observation is supported by [7] which notes that the most important variable that affects driver P-R time is driver expectation which can affect the P-R time by a factor of 2. There, the author concludes that an unexpected event can increase both the perception and reaction time of the driver. In addition, evidence in the psychology literature indicates that P-R time strongly depends on the type and intensity of stimulus [8]. Since IntelliDrive-enabled systems constitute a new type of stimulus with high intensity that help increase driver expectation, such systems are likely to shorten drivers' P-R times. In addition to assisted vehicles, partially or fully automated driving systems will be devised to further reduce the P-R time and hence the variation in responses. Here, as a control unit within the vehicle is in charge of the driving task, the variance in P-R time is essentially zero. This is because there is no human factor involved in this case. The mean of P-R time, however, is at its worst equal to the interval between the reception of two subsequent status packets from neighboring vehicles. Hence, the mean is dependent on the communications capacity within the network of vehicles and relies on different factors such as the number of nodes but is in general much less than the mean of the P-R time of assisted vehicles in the vast majority of traffic scenarios. As we shall see, lower P-R time would enable the assisted and automated vehicles to decrease their distance with their leaders without decreasing their speeds. This would lead to more compact clusters which consequently means higher flow of vehicles on the highway. IntelliDrive could also be utilized to help a vehicle perform more efficient lane-changing maneuvers. As we shall see, a lane change, which might at first seem unlikely for a driver, could be made possible with the help of
IntelliDrive. However, unlike its effect on driver P-R time, the effect of IntelliDrive on lane-changing is highly dependent on the specific traffic conditions.

In this paper, we propose a mathematical framework which can be used to foresee the effect of the gradual deployment of IntelliDrive-enabled vehicles on the efficiency of transportation systems. As a first step, we develop an appropriate mobility model which shall serve our analysis throughout the paper. For the base case of a single-lane highway, mathematical modeling of vehicle clusters is provided. To make the analysis more realistic, the modeling is generalized to multilane highways next. Appropriate lanechanging mechanisms are also discussed in the framework. With the mobility model in hand, we then proceed to derive mathematical expressions to address the effect of IntelliDrive on vehicular flow under mixed traffic conditions.

\section{Related Work}

The current literature on the safety and efficiency of vehicular networks contains studies conducted within both wireless communications and transportation societies. It has long been established that car clustering (platooning) in automated highways increases the highway capacity [9]. This is accomplished by automated vehicles following each other very closely at highway speeds and without increasing the risk of a collision [10,11]. Real-world experiments in this prospect have validated the increase in capacity [12]. However, stable clusters are viable only under a certain degree of intervehicle communications [13]. The safety and efficiency of manual and automated highways have been compared in [14]. There, automated highways with different levels of cooperation between individual vehicles and also platoon-based systems have been considered. Currently, due to practical and deployment cost considerations, the trend has moved from fully automated to driver-assisted vehicles. USDOT's IntelliDrive initiative is a pioneer in this prospect. The effect of driver-assisting technologies such as cruise control on vehicular traffic flow has been addressed in [15, 16]. In Adaptive Cruise Control (ACC) systems, the main objective is to safely increase driver comfort rather than improving highway capacity. The global impact of ACC on the safety of highways is studied in [17]. In that study, the effect of ACC on traffic flow capacity is considered a secondary issue. In [18], Tampere et al. prove that the time gap and driver relaxation times have the greatest effect on traffic stability. Based on this, they propose that any driver assistance system that intends to improve traffic stability should aim at influencing these parameters. Despite all this, there is a lack of a study that specifically addresses the effect of IntelliDrive on traffic flow efficiency.

Within the wireless communications society, specific characteristics of VANETs such as its highly dynamic topology, delay-sensitive applications, and constrained deployment region have led to the outgrowth of an abundant number of VANET-specific physical, MAC, and routing layer schemes $[4,19,20]$. The authors have also addressed MAC and network layer issues for urban deployments of VANETs 
[21]. The goal in most of these schemes is to establish reliable point-to-point communications between vehicles to achieve safety goals such as intersection collision warning. To the best of the authors' knowledge, there has little been done, if any, to study the effect of communications on traffic flow capacity and stability.

\section{System Modelling and Preliminaries}

3.1. Mobility Model: Vehicle Clustering. In this section, we study how vehicle clusters form and evolve in a multi-lane highway. Within a cluster, vehicles follow their leaders according to a car-following model elaborated in the next subsection. Furthermore, we shall see that the intervehicle spacings within a cluster ought to be enough to ensure a safe maneuver for the follower in case of the leaders abrupt braking. This spacing is a function of driver P-R time which is itself dependent on the level of IntelliDrive market penetration rate. Here, we first show, through a probabilistic analysis, how clusters are formed in a single lane highway. The analysis is then extended to account for multilane highways. As we shall later see, the beneficial effect of IntelliDrive on the capacity of multi-lane highways is not only because of its potential in reducing intervehicle spacings, but also due to the advantages it renders in terms of allowing for more efficient lane-changing operations.

3.1.1. Vehicle Clustering on a Single Lane. Consider a group of $N$ vehicles on a single-lane highway. Typically, each driver has a desired speed which it chooses from a (truncated) normal distribution $[22,23]$ within the range $\left[V_{\min } V_{\max }\right]$. Clearly, if a vehicle's desired speed is higher than at least one of the cars preceding it, it will join the cluster ahead; otherwise, it will trail back and form a new cluster. Hence, if the vehicles are numbered from 1 to $N$ from the beginning to the end of the road, the $i$ th vehicle would form a new cluster with probability $1 / i$ and join the cluster ahead with probability $(i-1) / i$. In what follows, we shall denote the initial randomly chosen speed of vehicle $i$ by $v_{i}$ and its ultimate speed within cluster $j$ by $V_{j}$. Note that all vehicles within a cluster move with the same speed. Within a cluster, a vehicle would leave a safety distance with its leader, proactively avoiding collisions.

Figure 1 shows the clustering probabilities for up to 5 vehicles. The numbers on the branches separated by commas show the length of the clusters, and the number in the circle to which the branch ends is the probability of having that specific clustering configuration. For example, the branch labeled as $(2,2$, and 1$)$ represents the configuration of having 2, 2 and 1 vehicles in the 1st, $2 \mathrm{nd}$, and $3 \mathrm{rd}$ clusters, respectively. As can be seen from the tree, this happens with the probability of $1 / 40$. The following example illustrates how these probabilities are derived. Assume that we have a $(2,2)$ configuration. The 5 th vehicle either joins the cluster ahead with probability $4 / 5$, hence arriving at a $(2,3)$ arrangement with probability $3 / 24 \times 4 / 5=1 / 10$, or trails back with probability $1 / 5$, resulting in the $(2,2,1)$ configuration with probability $3 / 24 \times 1 / 5=1 / 40$ (see dotted section of Figure 1). By extending this tree for an arbitrary number of vehicles, one can derive all the clustering probabilities. In this sense, each cluster can be seen as a kinematic wave [24] moving with the speed of its leader.

In what follows, we shall compute the average number of the clusters. Let us define $X_{i}$ as

$$
X_{i}= \begin{cases}1 & \text { if the } i \text { th vehicle is a clusterhead } \\ 0 & \text { otherwise }\end{cases}
$$

With this definition, it can be seen that if $C$ denotes the mean number of clusters

$$
\begin{aligned}
C & =E\left[\sum_{i=1}^{N} X_{i}\right]=\sum_{i=1}^{N} E\left[X_{i}\right] \\
& =\sum_{i=1}^{N} p_{i}=\sum_{i=1}^{N} \frac{1}{i}=1+\ln (N)(1+o(1)),
\end{aligned}
$$

where $p_{i}=P\left(X_{i}=1\right)$ and $o(1) \rightarrow 0$ as $N \rightarrow \infty$, where $N$ is the number of vehicles on the road.

3.1.2. Multilane Highway System. Here, we study the extension of the above scenario to multi-lane highways. Multi-lane highways typically allow for a more dynamic environment where vehicles with a high desired speed that are "stuck" in a low-speed cluster of vehicles are allowed to change lanes and join a higher-speed cluster on the other lane. As mentioned before, a vehicle on a specific lane either moves with its own desired speed (as a cluster head) or with the speed of its predecessor, whichever is lower. However, in this case, if the current speed of a vehicle within a cluster is lower than its desired speed, it might have the chance to overtake this cluster of vehicles and join a higher-speed one on the lane to its left. To this end, assume vehicle $i$ with the desired speed $v_{i}$ resides in cluster $m$ (and hence has speed $V_{m}$ ) when cluster $n$ on the lane to its left with speed $V_{n}$ comes cruising by. Here, vehicle $i$ decides to make a lane change and join cluster $n$ if $v_{i} \geq V_{n}>V_{m}$. Once such a vehicle has made a lane-change decision, it seeks an opportunity to merge into the higher-speed lane by searching for a sizeable gap. Such a gap-acceptance model works as follows. Consider cluster $n+1$ to immediately follow cluster $n$ on the left lane. We assume that in equilibrium conditions, the speed of a leading cluster is larger than the trailing one (because otherwise they would ultimately merge into a single cluster) in each lane. This fact is well anticipated by our clustering model proposed in Section 3.1.1. At the same time, we also assume that the left lane clusters have higher speeds than all the ones on the right lane. Hence, under these conditions, we have $V_{m}<$ $V_{n+1}<V_{n}$. A valid gap-acceptance model should determine the circumstances under which vehicle $i$ is able to make a lane change and join cluster $n$ such that it collides with neither the vehicles of cluster $n$ nor those of cluster $n+1$. Note that after making a lane change and with an initial speed of $V_{m}$, vehicle $i$ would accelerate to reach its final speed, $V_{n}$. Since $V_{m}<V_{n+1}$, it is during this period of time that the trailing cluster $n+1$ could collide with vehicle $i$. Assuming the worst 


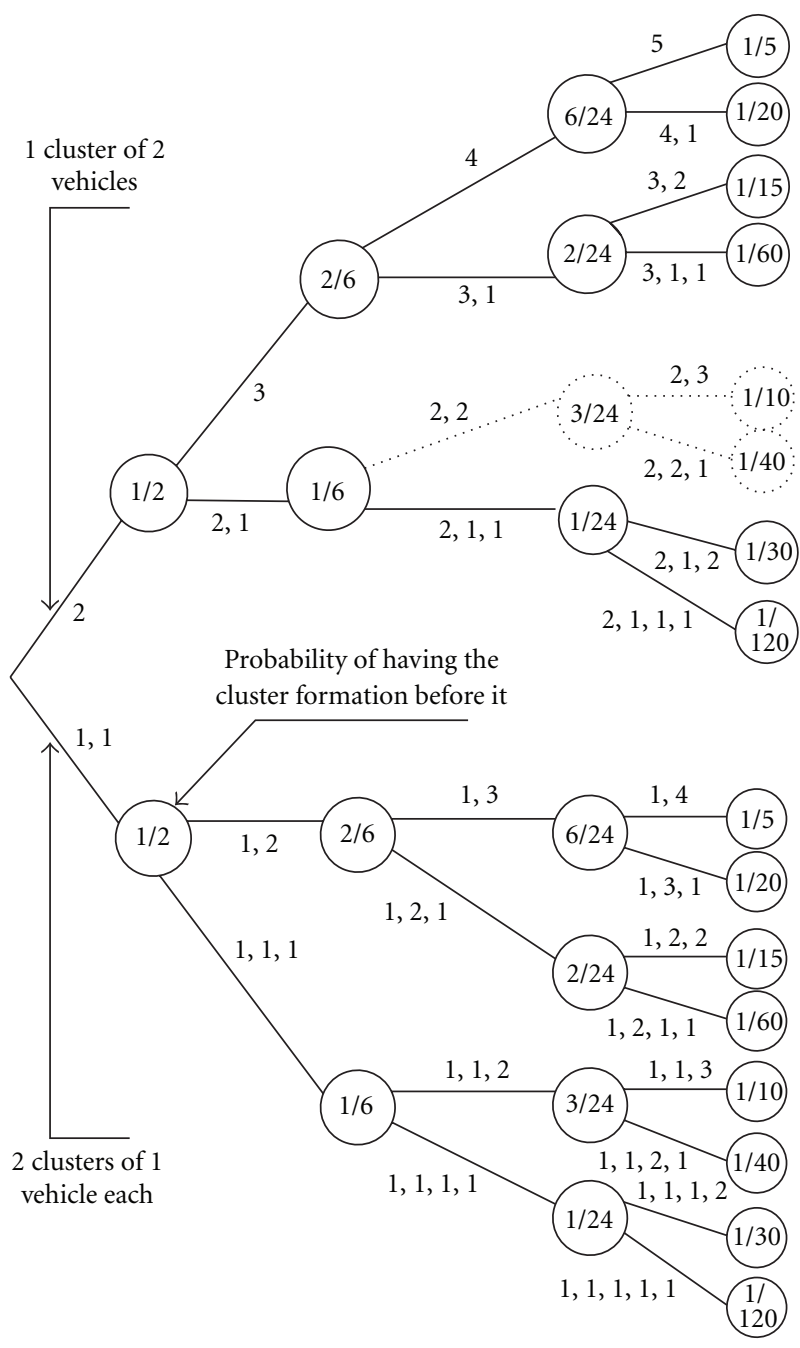

Figure 1: Decision tree demonstrating clustering probabilities.

case where the vehicles in cluster $n+1$ do not decelerate (and hence continue cruising with their previous speeds) upon observing a leading lane changing vehicle, it can be verified that the following condition ensures a safe lane-changing maneuver for vehicle $i$ :

$$
s_{n, n+1}>\frac{\left(V_{n+1}-V_{m}\right)^{2}-\left(V_{n}-V_{n+1}\right)^{2}}{2 a_{i}},
$$

where $s_{n, n+1}$ is the spacing between clusters $n$ and $n+1$ at the intended time of the lane change and $a_{i}$ denotes vehicle $i$ comfortable acceleration rate. The derivation of (3) is elaborated in Appendix A.

A driver of a unequipped vehicle has to rely on his own visual eyesight and estimate of the speed and spacing in order to make a lane-change decision. Moreover, in the case where it cannot see a possible trailing vehicle in the other lane in its rear or side mirrors (e.g., when there is curvature in highway trajectory or fluctuations in its elevation), it should account for the worst case when a high-speed vehicle rapidly closes up onto it once the lane change has been made. An IntelliDriveenabled vehicle, on the other hand, typically knows the exact location and speed of a trailing vehicle on the destination lane in case the latter is also assisted. Hence, the driver does not have to assume for the worst case scenario and further would not suffer from estimation deficiencies to miss a potential lane-change opportunity. Here, we want to study the effect of IntelliDrive on the average number of vehicles that leave cluster $m$ for the higher speed cluster $n$. In general, this value is dependent on the relative speeds and the distance between the leading and trailing clusters on the destined lane, that is, even if all the vehicles in cluster $m$ were IntelliDriveenabled, none of them could perform a lane change when $s_{n, n+1}$ does not satisfy (3). Hence, the effect of IntelliDrive on the lane-change maneuvers entirely depends on the real-time traffic conditions. Moreover, cluster $m$ could belong to one of the following categories with respect to cluster $n$ :

(1) neither the equipped nor the unequipped vehicles within it are able to join cluster $n$,

(2) only the equipped vehicles, based on the real time information that they obtain, are able to join cluster $n$

(3) all vehicles, whether equipped or not, can complete the lane-change maneuver and join cluster $n$.

The road topology along with the gap-acceptance equation (3) determines to which of the above classes does cluster $m$ belong. For an equipped vehicle, equation (3) suffices to indicate whether a lane change is possible. For unequipped vehicles, road topology plays a role as well, that is, if the road topology is such that any trailing cluster is visible to the lanechanging vehicle, it would deploy (3) to estimate whether it could perform the lane change or not. If not, it should assume that the trailing cluster has speed $V_{n+1}=V_{\max }$ and resides at the furthest visible point from cluster $n$. For future analysis, let $Y_{m n}=j$ if cluster $m$ is category $j \in\{1,2,3\}$ with respect to cluster $n$.

To see how many vehicles leave cluster $m$ for cluster $n$, we define $X_{m n}(i)$ as

$$
X_{m n}(i)= \begin{cases}1 & \text { vehicle } i \text { in cluster } m \text { leaves for cluster } n \\ 0 & \text { otherwise }\end{cases}
$$

Note that $X_{m n}(i)=1$ if $v_{i} \geq V_{n}$ and otherwise vehicle $i$ stays in cluster $m$. With the above definition, $X_{m n}=$ $\sum_{i=1}^{N_{m}} X_{m n}(i)$ denotes the total number of departures from cluster $m$ for cluster $n$ where $N_{m}$ is the number of vehicles within cluster $m$.

3.2. Mobility Model: Car Following. The spacing between vehicles within a cluster should allow for a safe and comfortable declaration of the follower in case of the leader's sudden braking. As we shall see, this spacing is highly dependent on the driver's P-R time which is assumed to be a function of IntelliDrive market penetration rate. Car following models have long been studied by transportation engineers. Models such as Pipes [25], General Motors (GM) [26, 27], and Gipps [28] account for different car following behaviors. 
Our analysis adopts a variation of the Gipps' model which is rather conservative. In this model, vehicles allow for a safety distance between themselves and their leading vehicle. The safety distance should be such that in the event when the leader (vehicle $i-1$ ) applies a sudden brake and slows down with maximum deceleration $B_{i-1}$, the follower (vehicle i) should be able to safely stop behind it after going through a P-R process and a deceleration process at a comfortable rate $b_{i}\left(\left|b_{i}\right|<\left|B_{i}\right|\right)$. If we denote by $x_{i}$ and $v_{i}$ the position and speed of the $i$ th vehicle, and by $x_{i-1}^{*}$ and $x_{i}^{*}$ the stopping position of the leader and follower, respectively, we shall have

$$
\begin{aligned}
& x_{i-1}^{*}=x_{i-1}(t)-\frac{v_{i-1}^{2}(t)}{2 B_{i-1}}, \\
& x_{i}^{*}=x_{i}(t)+v_{i} \tau_{i}-\frac{v_{i}^{2}(t)}{2 b_{i}},
\end{aligned}
$$

where $\tau_{i}$ is driver $i$ 's P-R time. Equation (6) is true because the follower would go through a P-R time before applying the brakes. To ensure safety, we must have $x_{i-1}^{*}-L_{i-1} \geq x_{i}^{*}$ where $L_{i-1}$ is the length of the $(i-1)$ th vehicle. Within a cluster, where the two vehicles have the same speed $v=v_{i}=v_{i-1}$, we have for the intervehicle spacing, $S_{i}$

$$
S_{i}=x_{i-1}-x_{i} \geq \tau_{i} v+G v^{2}+L,
$$

where $G=1 / 2 B-1 / 2 b$ and the subscripts for $B, b$, and $L$ have been dropped for simplicity. As evident from (7), the stochastic properties of the intervehicle spacing is solely dependent on the stochastic properties of driver P-R time. As discussed before, a driver of an unequipped vehicle on average goes through a longer P-R time in case of an unexpected situation in comparison to an assisted vehicle where the driver has been alerted (and hence his or her P$\mathrm{R}$ time reduced) via timely warning messages. In [7], the author emphasizes that driver attention is a graded function. Hence, the above two cases represent a graded continuum rather than a dichotomy. Based on this and prior discussions, we propose that the mean and the variance of drivers' P-R time is a decreasing linear function of IntelliDrive market penetration rate, that is, the more the percentage of assisted vehicles within a cluster, the lower is the mean and variance of its drivers' P-R times. Note that the linear relation is considered for the convenience to expedite the mathematical analysis and that other decreasing functions would yield similar results of this paper. Moreover, if $\alpha$ denotes the market penetration rate of assisted vehicles, we assume that the driver P-R time, $\tau(\alpha)$ has a truncated normal distribution with (The normal distribution for driver P-R time is justified in [7], noting that in reality it is mostly skewed towards larger values.)

$$
\begin{aligned}
& \mu_{\tau(\alpha)}=\alpha\left(\mu_{\tau(\alpha=1)}-\mu_{\tau(\alpha=0)}\right)+\mu_{\tau(\alpha=0)}, \\
& \sigma_{\tau(\alpha)}^{2}=\alpha\left(\sigma_{\tau(\alpha=1)}^{2}-\sigma_{\tau(\alpha=0)}^{2}\right)+\sigma_{\tau(\alpha=0)}^{2}
\end{aligned}
$$

where $\mu$ and $\sigma$ are the mean and variance of driver P-R time with $\mu_{\tau(\alpha=1)} \leq \mu_{\tau(\alpha=0)}$ and $\sigma_{\tau(\alpha=1)}^{2} \leq \sigma_{\tau(\alpha=0)}^{2}$. This way, the spacing between an assisted vehicle and its leader also follows a truncated normal distribution with the same variance as in (9) and mean an appropriately scaled and shifted version of (8). The P-R time of unequipped vehicles is the same as when $\alpha=0$ for assisted vehicles and the P-R time for automated vehicles is deterministic (and equal to $\mu_{\tau(\beta)}$ ) as discussed before. Figure 2 shows how the probability distributions of driver's P-R time varies with IntelliDrive market penetration. As we shall see in the next section, the mean values for driver P-R times suffice to compute the lower bounds on the expected value of vehicular flow.

\section{Effect of IntelliDrive on Highway Efficiency}

In this section, we quantify the enhancements IntelliDrive renders in terms of traffic flow efficiency. Moreover, we mathematically explore the extent at which IntelliDrive can increase the flow of vehicles on roads without increasing the risk of collisions. As mentioned before, this is a result of the drivers' reduced P-R time which lets them maintain their speed while reducing their spacing with the leading vehicle. We shall also study how IntelliDrive can enhance lanechange maneuvers in multilane highways. In what follows, the term flow shall denote the number of vehicles that pass an arbitrary point of the highway in unit time. In equilibrium conditions, the following relation exists between traffic flow characteristics, flow $(q)$, density $(k)$, and speed $(v)$ [29]:

$$
q=k v
$$

As vehicles within a cluster move with the same speed, equation (10) can be used as follows to derive the flow within cluster $i$ as:

$$
q_{i}=\frac{N_{i} V_{i}}{\sum_{j=1}^{N_{i}} S_{i j}}
$$

where $V_{i}, N_{i}$, and $S_{i j}$ are the speed, number of vehicles, and intervehicle spacings in cluster $i$. Note that here, flow is a probabilistic value as a result of the intervehicle spacings being probabilistic due to (7). The following theorem addresses its expected value.

Theorem 1. For a single cluster of $N_{i}$ vehicles all moving with the same speed $V_{i}$, the following holds for the flow of the cluster when each vehicle within it is assisted with probability $\alpha$ and automated with probability $\beta$ :

$$
E\left[q_{i}\right] \geq \frac{V_{i}}{c+a \beta+b \alpha^{2}(1-\beta)},
$$

where $a=V_{i}\left(\mu_{\tau(\beta)}-\mu_{\tau(\alpha=0)}\right), b=V_{i}\left(\mu_{\tau(\alpha=1)}-\mu_{\tau(\alpha=0)}\right)$, and $c=G V_{i}^{2}+\mu_{\tau(\alpha=0)} V_{i}+L$.

Proof. See Appendix B.

Notice that the lower bound is independent of the number of vehicles within the cluster, $N_{i}$. We shall later see that the lower bound is also quite tight. Figure 3 depicts the enhancement in flow due to IntelliDrive. The values used to evaluate (12) are gathered in Table 1. First, notice that, as 
TABle 1: Parameter values.

\begin{tabular}{lccccccccccc}
\hline$\mu_{\tau(\alpha=0)}$ & $2 \mathrm{~s}$ & $\mathrm{~B}$ & $-6 \frac{m}{s^{2}}$ & $\bar{\alpha}_{1}^{r}$ & $27.3 \%$ & $\bar{\beta}_{1}^{r}$ & $9.1 \%$ & $N_{1}^{r}$ & 30 & $E\left[\bar{N}_{1}^{r}\right]$ & 28.3 \\
\hline$\mu_{\tau(\alpha=1)}$ & $1 \mathrm{~s}$ & $\mathrm{~b}$ & $-3 \frac{m}{s^{2}}$ & $\bar{\alpha}_{1}^{l}$ & $36.6 \%$ & $\bar{\beta}_{1}^{l}$ & $12.2 \%$ & $N_{1}^{l}$ & 10 & $E\left[\bar{N}_{1}^{l}\right]$ & 11.7 \\
\hline$\mu_{\tau(\beta)}$ & $0.1 \mathrm{~s}$ & $\alpha$ & $30 \%$ & $\bar{\alpha}_{2}^{l}$ & $30 \%$ & $\bar{\beta}_{2}^{l}$ & $10 \%$ & $N_{2}^{l}$ & 15 & $E\left[\bar{N}_{2}^{l}\right]$ & 15 \\
\hline$L$ & $5 \mathrm{~m}$ & $\beta$ & $10 \%$ & $\bar{\alpha}_{3}^{l}$ & $29 \%$ & $\bar{\beta}_{3}^{l}$ & $9.7 \%$ & $N_{3}^{l}$ & 20 & $E\left[\bar{N}_{3}^{l}\right]$ & 31.8 \\
\hline
\end{tabular}

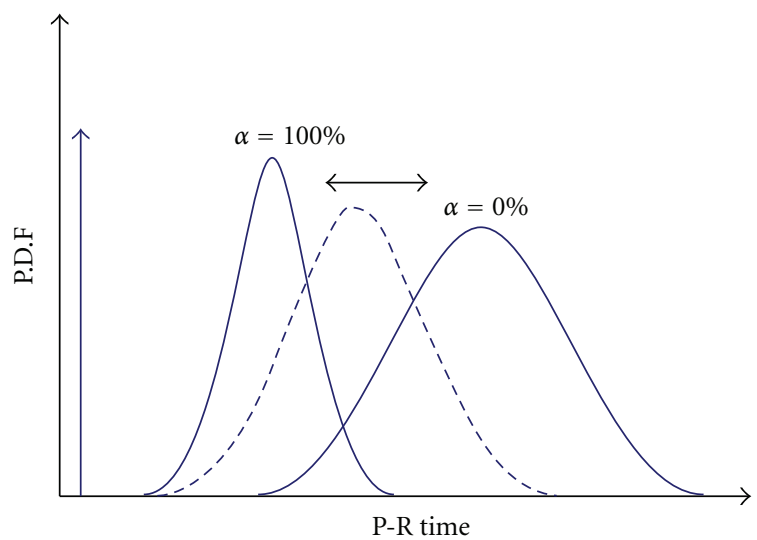

Figure 2: Perception-Reaction times for different classes of vehicles. The normal distributions correspond to the P-R times of assisted vehicles for various market penetration rates. P-R time of unequipped vehicles is the same as when $\alpha=0$. The delta function represents the P-R time of an automated vehicle.

seen in Figure 3(a), the flow-speed relation in a cluster of vehicles moving with the same speed follows closely the trend of a typical macroscopic flow-speed relation in a highway [29]. The only difference is that the speed at which the peak flow occurs is somewhat lower which is due to the rather conservative car following model we have used in our analysis. Hence, our results also provide a good insight on the effect of IntelliDrive on the macroscopic behaviors of general highway systems. Figure 3(a) also shows the effect of IntelliDrive on the speed-flow curve for various penetration rates of assisted and automated vehicles. Further, by looking at Figure 3(c), one can see that for a fixed $\beta$, flow increases by increasing $\alpha$. The same is true when $\beta$ is increased for a fixed $\alpha$ (see Figure 3(d)). However, the augmentation in throughput obtained by increasing $\beta$ is greater than the one achieved by increasing $\alpha$. This is seemingly right since automated vehicles have lower P-R times than assisted vehicles. Moreover, when all vehicles are automated, flow can increase by up to $65 \%$, whereas when they are all just assisted, the increase in flow is by about $25 \%$. As a final remark, in Figure 3(d), we have compared the exact value of flow against the lower bound in (12). As can be seen, with an accuracy of about $99.9 \%$, the lower bound serves as a perfect approximation for the exact value.

For a two-lane highway, consider an instance where $N^{r}$ and $N^{l}$ vehicles initially reside on the right and left lanes, respectively, $\left(N^{r}+N^{l}=N\right)$. We number the clusters from 1 through $C^{r}$ and 1 through $C^{l}$ on the right and left lanes, from the beginning to the end of each lane. Hence, $N^{r}=\sum_{i=1}^{C_{r}} N_{i}^{r}$ and $N^{l}=\sum_{i=1}^{C_{l}} N_{i}^{l}$, where $N_{i}^{r}\left(N_{i}^{l}\right)$ is the number of vehicles in cluster $i$ on the right (left) lane. Here, we address the average traffic flow after a group of high-speed clusters on the left lane overtake a group of lower speed ones on the right lane, during which lane-changing maneuvers can happen when needed and allowed based on the discussion in Section 3.1.2. As we shall see, the effect of IntelliDrive on traffic flow is due to its role in the lane-changing maneuvers as well as the lowering of driver P-R times. Note that if $C=C^{r}+C^{l}$, then according to (2) we would have $C=O(\ln N)$. Hence, the effect of the bounded inter-cluster spacings on the total flow would be negligible as $N$ grows arbitrarily large, and a weighted average of the per-cluster flows would serve as a fine approximation of the total flow. The total flow after the overtakings take place can be stated as (the superscripts have been dropped for simplicity)

$$
q=\frac{\sum_{i=1}^{C} N_{i} q_{\mathrm{i}}}{N}
$$

Hence,

$$
\begin{aligned}
E[q] & =\frac{\sum_{i=1}^{C} E\left[N_{i} q_{i}\right]}{N} \\
& =\frac{1}{N} \sum_{i=1}^{C} \sum_{n_{i}=1}^{N} n_{i} E\left[q_{i} \mid N_{i}=n_{i}\right] P\left(N_{i}=n_{i}\right) \\
& \stackrel{(1)}{\geq} \frac{1}{N} \sum_{i=1}^{C} E\left[q_{i}\right] \sum_{n_{i}=1}^{N} n_{i} P\left(N_{i}=n_{i}\right) \\
& =\frac{1}{N} \sum_{i=1}^{C} E\left[q_{i}\right] E\left[N_{i}\right],
\end{aligned}
$$

where (1) is due to Theorem 1 which implies that the lower bound on the expected flow of a cluster is independent of the number of vehicles within it.

In what follows, we aim at comparing the value of $E\left[q_{i}\right]$ after the overtakings take place with and without the utilization of IntelliDrive. When IntelliDrive is available, we assume that the penetration rate of assisted and automated vehicles in each cluster is initially $\alpha$ and $\beta$ before the overtakings take place. In order to compute (14), we need to have the ultimate values for $E\left[q_{i}\right]$ 's and $E\left[N_{i}\right]$ 's after the overtakings take place. For $E\left[q_{i}\right]$, note that, according to (12), the only influential parameters on flow that change during the course of the overtakings are the percentage of equipped vehicles in each cluster. Here, we study their evolution over time.

For an arbitrary cluster $m$ on the right and $n$ on the left lane, let $n_{m}^{\prime}=\max _{Y_{m i} \in\{2,3\}} i \in\{1, \ldots, n-1\}$ and $n_{m}^{\prime \prime}=$ 
$\max _{Y_{m i}=3} i \in\{1, \ldots, n-1\}$ where again, $Y_{m n}=j$ if cluster $m$ is category $j \in\{1,2,3\}$ with respect to cluster $n$. This way, $V_{n_{m}^{\prime}}^{l}$ is the speed of the nearest leading cluster $n_{m}^{\prime}$ which the equipped vehicles in cluster $m$ have had the chance to join (hence $m$ is category 2 or 3 with respect to $n_{m}^{\prime}$ ). If no such cluster exists, then $V_{n^{\prime}}^{l}=V_{\max }$. On the other hand, $V_{n_{m}^{\prime \prime}}^{l}$ is the speed of the nearest leading cluster $n_{m}^{\prime \prime}$ which all vehicles in cluster $m$, whether equipped or not, have had the chance to join. Again, if no such cluster exists, we have $V_{n_{m}^{\prime \prime}}^{l}=V_{\max }$. Note that essentially $V_{n_{m}^{\prime}}^{l} \leq V_{n_{m}^{\prime \prime}}^{l}$. With this setting, if $\alpha_{m_{n+1}}^{r}$ denotes the probability that a vehicle within cluster $m$ on the right lane is assisted, right before being overtaken by cluster $n+1$ on the left lane, we have, starting with $\alpha_{m_{1}}^{r}=\alpha$

$$
\alpha_{m_{n+1}}^{r}=\left\{\begin{array}{c}
\alpha_{m_{n}}^{r}, \quad Y_{m n}=1, \\
\frac{\alpha_{m_{n}}^{r} P\left(v<V_{n}^{l} \mid V_{m}^{l} \leq v<V_{n_{m}^{\prime}}^{l}\right)}{1-\left(\alpha_{m_{n}}^{r}+\beta_{m_{n}}^{r}\right)\left(1-P\left(v<V_{n}^{l} \mid V_{m}^{r} \leq v<V_{n_{m}^{\prime}}^{l}\right)\right)}, \\
Y_{m n}=2, \\
\frac{\alpha_{m_{n}}^{r} P\left(V_{m}^{r} \leq v<V_{n_{m}^{\prime \prime}}^{l}\right)}{\left(\alpha_{m_{n}}^{r}+\beta_{m_{n}}^{r}\right) P\left(V_{n_{m}^{\prime}}^{l} \leq v<V_{n_{m}^{\prime \prime}}^{l}\right)+P\left(V_{m}^{r} \leq v<V_{n_{m}^{\prime}}^{l}\right)}, \\
Y_{m n}=3 .
\end{array}\right.
$$

The derivation of (15) is provided in Appendices A and B. Through (15), the ultimate probability that a vehicle in cluster $m$ on the right lane is assisted, $\bar{\alpha}_{m}^{r}$, can be derived by computing $\alpha_{m_{C_{l+1}}}^{r}$. The same as above holds for automated vehicles by only replacing $\beta$ for $\alpha$ in (15). With the ultimate values of $\alpha$ and $\beta$ in hand, the new values of $E\left[q_{i}\right]$ 's can be derived from (12).

Let us now consider the evolution of the $E\left[N_{i}\right]$ 's. For that, we compute the expected number of vehicles that leave a cluster for another. Note that for an arbitrary cluster $m$ on the right lane, one of the following is true with respect to an arbitrary cluster $n$ on the left lane. (Here, we assume that a vehicle surely performs the lane-change maneuver once it knows it is safe to do so. In the general case for assisted drivers, human uncertainty can be accounted for by incorporating an appropriate probability function based on which the assisted driver makes a lane-change decision.)

(1) When neither the equipped nor the unequipped vehicles leave cluster $m$ for cluster $n$ we have $X_{m n}=0$.

(2) When just the equipped vehicles in $m$ can join cluster $n$

$$
\begin{aligned}
E\left[X_{m n}(i)\right] & \stackrel{(1)}{=} P\left(X_{m n}(i)=1\right) \\
& =\left(\beta_{m_{n}}^{r}+\alpha_{m_{n}}^{r}\right) P\left(v_{i} \geq V_{n}^{l} \mid V_{n_{m}^{\prime}}^{l} \geq v_{i} \geq V_{m}^{r}\right),
\end{aligned}
$$

where (1) is due to (4). Let us elaborate on the condition of the probability. Note that for vehicle $i$ in cluster $m$ we already know $v_{i} \geq V_{m}$. Also, if it is equipped and still resides in cluster $m$ at the the time it encounters cluster $n$ on the left lane, we know that $v_{i} \leq V_{n_{m}^{\prime}}^{l}$.
(3) When equipped and unequipped vehicles can merge from $m$ into $n$, we have:

$$
\begin{aligned}
& E\left[X_{m n}(i)\right] \\
& =\left(\beta_{m_{n}}^{r}+\alpha_{m_{n}}^{r}\right) P\left(v_{i} \geq V_{n}^{l} \mid V_{n_{m}^{\prime}}^{l} \geq v_{i} \geq V_{m}^{r}\right) \\
& \quad+\left(1-\beta_{m_{n}}^{r}-\alpha_{m_{n}}^{r}\right) P\left(v_{i} \geq V_{n}^{l} \mid V_{n_{m}^{\prime \prime}}^{l} \geq v_{i} \geq V_{m}^{r}\right) .
\end{aligned}
$$

Here, at the time vehicle $i$ in cluster $m$ encounters cluster $n$ on the left lane it has $v_{i} \leq V_{n_{m}^{\prime}}^{l}$ if it is equipped and has $v_{i} \leq V_{n_{m}^{\prime \prime}}^{l}$ if it is unequipped.

For all the above scenarios, we have

$$
\begin{aligned}
E\left[X_{m n}\right] & =E\left[\sum_{i=1}^{N_{m_{n}}^{r}} X_{m n}(i)\right] \\
& =E\left[N_{m_{n}}^{r}\right] E\left[X_{m n}(i)\right],
\end{aligned}
$$

where $N_{m_{n}}^{r}$ is the number of vehicles within cluster $m$ on the right lane, just before the $n$th cluster on the left lane reaches it. Here,

$$
E\left[N_{m_{n}}^{r}\right]=E\left[N_{m}^{r}\right]-\sum_{i=1}^{n-1} E\left[X_{m i}\right]
$$

where $N_{m}^{r}=N_{m_{1}}^{r}$. Note that (18) follows from Wald's equation. (Wald's equation states that $E\left[\sum_{i=1}^{T} X_{i}\right]=E[T] E[X]$ where the $X_{i}$ 's are i.i.d, and independent from $T$ and $E[T], E[x]<\infty$.) The use of the latter equation needs independent and identically distributed (i.i.d) $X_{m n}$ (i)'s which is true as the desired speed of a vehicle and the chances of it being equipped are independent of any other. The other conditions that need to be true in order to let us use the Wald's equation is for $N_{m_{n}}^{r}$ and $X_{m n}(i)$ to have finite mean values and also to be independent which all clearly hold in our case.

Finally, (16) or (17) are used along with (18) and (19) to give us the expected number of vehicles that leave a cluster for another and consequently the ultimate number of vehicles within each cluster. The final percentage of assisted vehicles for cluster $n$ on the left lane is obtained as

$\bar{\alpha}_{n}^{l}$

$$
=\frac{\alpha N_{n}^{l}+\sum_{m: Y_{m n}=3} \alpha_{m_{n}}^{r} X_{m n}+\sum_{m: Y_{m n}=2}\left(\alpha_{m_{n}}^{r} /\left(\alpha_{m_{n}}^{r}+\beta_{m_{n}}^{r}\right)\right) X_{m n}}{N_{n}^{l}+\sum_{m} X_{m n}} .
$$

The numerator shows the expected number of assisted vehicles. Here, $\alpha N_{n}^{l}$ is the initial number of assisted vehicles cluster $n$ contains before the overtakings take place. The second and third terms represent the contribution of category 2 and 3 clusters during the course of the overtakings. Also, the denominator is the total number of vehicles in cluster $n$ upon the completion of the overtakings. A similar equation is true for $\bar{\beta}_{n}^{l}$ by just replacing $\beta$ for $\alpha$. 


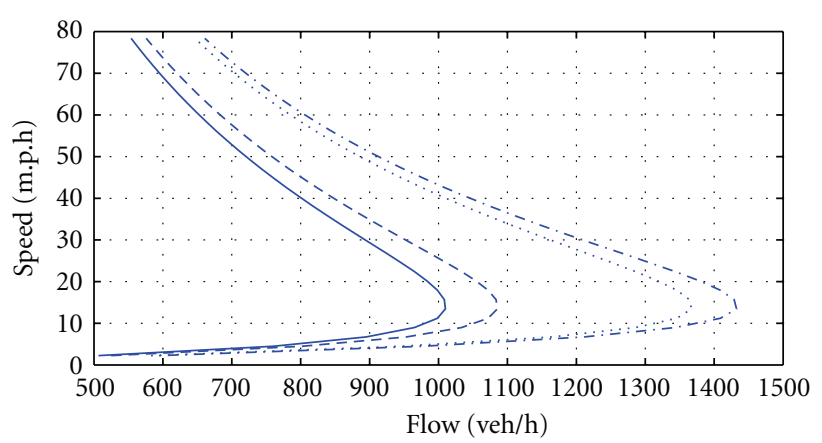

$$
\begin{aligned}
& -\alpha=0 \%, \beta=0 \% \quad \cdots \cdots \alpha=0 \%, \beta=50 \% \\
& \text {-- } \alpha=50 \%, \beta=0 \% \quad \text {..- } \alpha=50 \%, \beta=50 \%
\end{aligned}
$$

(a)

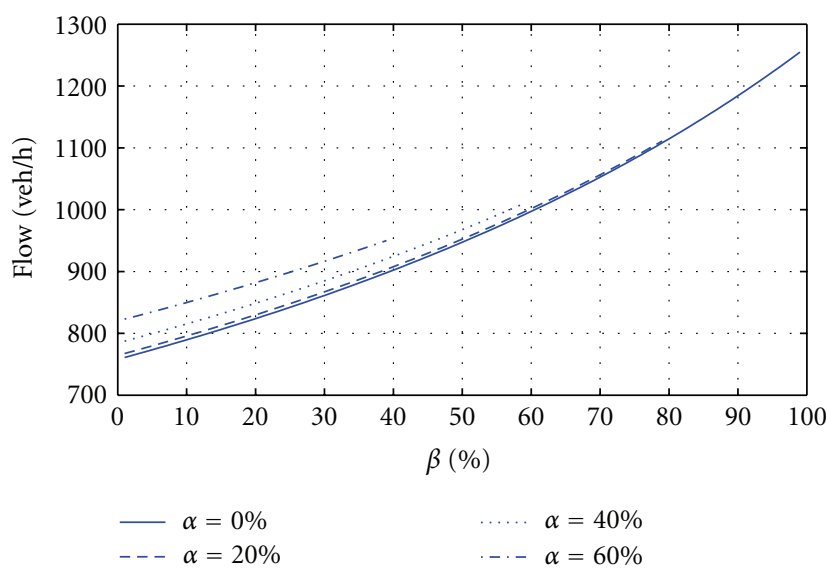

(c)

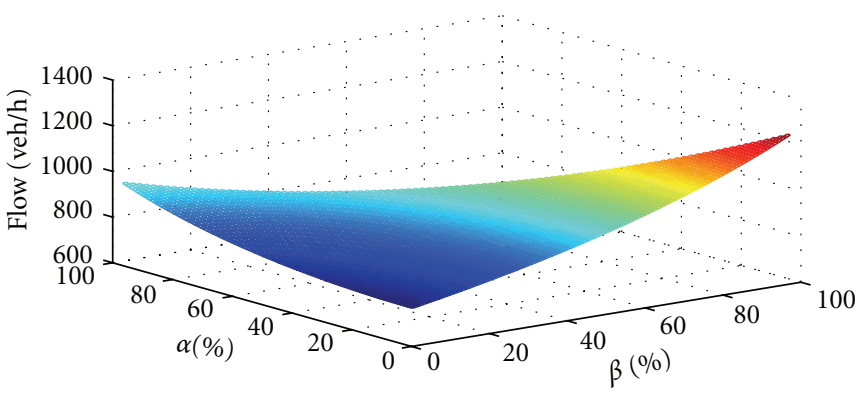

(b)

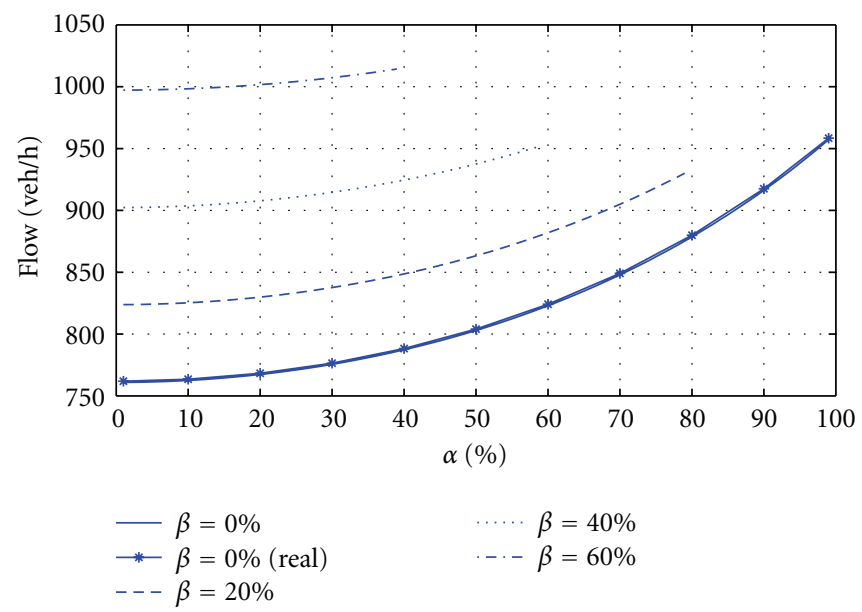

(d)

Figure 3: (a) The effect of IntelliDrive on the flow-speed curve. (b) Traffic flow variation with the penetration of automated and assisted vehicles into the market. (c) Flow enhancement for a fixed penetration of assisted vehicles. (d) Flow enhancement for a fixed penetration of automated vehicles. $V=44.8$ m.p.h. in (b), (c), and (d).

To clarify the concept, here we consider an example scenario of a two-lane highway depicted in Figure 4, where three clusters on the left lane overtake a lower speed one on the right lane. As can be seen from Figure 4(b), the cluster on the right lane is category 2 with respect to the first cluster on the left lane, that is, $Y_{11}=2$. This is because if vehicle $i$ on the right lane that has made a lane-change decision (since $\left.v_{i} \geq V_{1}^{l}\right)$ is equipped, then it is able to figure out that the current gap between the first and second clusters on the left lane, that is, $\mathrm{s}_{12}$, is more than the minimum required $(100 \mathrm{~m})$ anticipated by the gap-acceptance equation (3). Hence, it is able to safely make a lane change. However, if it is not equipped, it goes on to assume that the second cluster resides at the furthest point which has line of sight to the first and has virtually the same speed as $V_{1}^{l}$. According to (3), this requires a gap more than $150 \mathrm{~m}$. But since the furthest lineof-sight point is only 80 meters away, lane-changing cannot be performed by an unequipped vehicle. By (15) and letting $m=1$ and $n=1, \alpha_{1_{2}}^{r}$ can be computed by noting that $\alpha_{1_{1}}^{r}=\alpha$ and $V_{1_{1}^{\prime}}^{l}=V_{\max }$. Further, we have according to (18) and (16) that $E\left[X_{11}\right]=N_{1}^{r}(\alpha+\beta) P\left(v_{i} \geq V_{1}^{l} \mid V_{\max } \geq v_{i} \geq V_{1}^{r}\right)$ and hence according to (19), $E\left[N_{1_{2}}^{r}\right]=N_{1}^{r}-E\left[X_{11}\right]$.

Following on, note that the cluster on the right lane is category 1 with respect to the second cluster on the left lane hence, $\alpha_{1_{3}}^{r}=\alpha_{1_{2}}^{r}$ and $X_{12}=0$. Thus the market penetration of the equipped vehicles and the total number of vehicles within the second cluster remain unchanged when it overtakes the cluster on the right lane.

Finally, as we have $Y_{13}=3$, the ultimate penetration rate of the equipped vehicles in the cluster on the right lane can be obtained by letting $m=1$ and $n=3$ in (15) which results in $\bar{\alpha}_{1}^{r}=\alpha_{1_{4}}^{r}=\alpha_{1_{3}}^{r}$ since $V_{3_{1}^{\prime \prime}}^{l}=V_{3_{1}^{\prime}}^{l}=V_{1}^{l}$. The ultimate number of vehicles in this cluster is then $N_{1}^{r}-E\left[X_{11}\right]-E\left[X_{13}\right]$, where $E\left[X_{13}\right]=N_{1_{3}}^{r} P\left(v_{i} \geq V_{3}^{l} \mid V_{1}^{l} \geq v_{i} \geq V_{1}^{r}\right)$ due to (17) and further (18). Also, for the ultimate penetration rate of equipped vehicles on the left lane, we have due to (20) that $\bar{\alpha}_{1}^{l}=\left(\alpha N_{1}^{l}+(\alpha /(\alpha+\beta)) E\left[X_{11}\right]\right) /\left(N_{1}^{l}+E\left[X_{11}\right]\right), \bar{\alpha}_{2}^{l}=\alpha$ and $\bar{\alpha}_{2}^{l}=\alpha N_{3}^{l}+\alpha_{13}^{r} E\left[X_{13}\right] / N_{3}^{l}+E\left[X_{13}\right]$. 


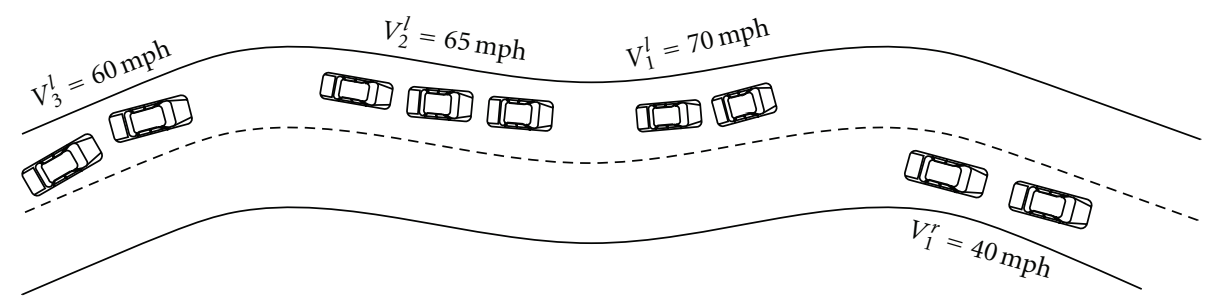

(a)

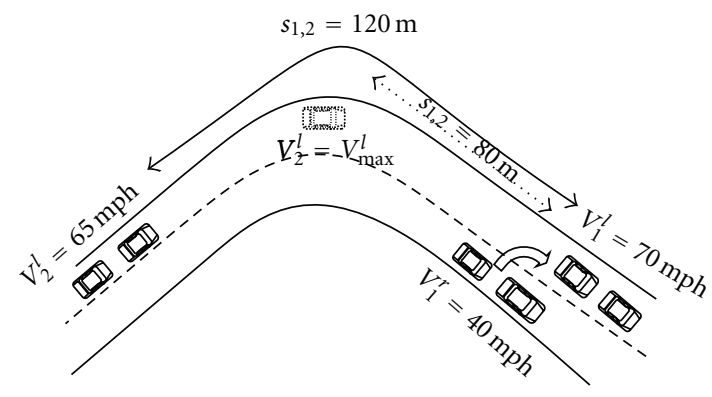

(b)

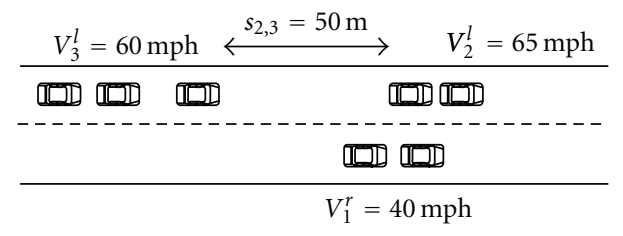

(c)

Figure 4: (a) Two-lane highway. The distribution of the desired speed of the vehicles is truncated normal with $\mu=60, \sigma=8$ within the [40-80] m.p.h range. (b) Cluster on the right lane is category 2 with respect to the first cluster on the left. (c) Cluster on the right lane is category 1 with respect to the second cluster on the left.

Table 1 exhibits the ultimate values of IntelliDrive market penetration rates and the number of vehicles within each cluster. These values are used in conjunction with (12) to evaluate the average flow over all clusters in (14). When there is no IntelliDrive, we have $X_{11}=X_{12}=0$ and $E\left[X_{13}\right]=$ $N_{1}^{r} P\left(v_{i} \geq V_{3}^{l} \mid V_{\max } \geq v_{i} \geq V_{1}^{r}\right)$. For our example scenario (with the IntelliDrive market penetration rates of $\alpha=30 \%$ and $\beta=10 \%$ ), the total flow after the overtakings take place is higher than when there is no IntelliDrive deployment (702 as opposed to $672 \mathrm{veh} / \mathrm{h}$ ).

Notice that the effect of IntelliDrive on the flow of a multi-lane highway is due to two main factors. One, as before, is by lowering the P-R time of drivers which leads to a higher per-cluster flow. The other means by which IntelliDrive can affect flow is by letting the equipped vehicles make lane changes that are not typically allowed for the unequipped vehicles. Notice that this does not necessarily increase the total flow even if the lane-changing vehicle joins a higher-speed cluster than its own. This is evident from the flow-speed curve in Figure 3 which implies that higher speed does not necessarily correspond to higher flow. Hence, to assess the effect of IntelliDrive on the total flow, the following should be determined. If there is no category 2 cluster on the right lane, the effect of IntelliDrive on flow is always constructive. For any category 2 cluster, on the other hand, we should check whether the flow corresponding to its speed is higher or lower than the flow of the cluster to which the lane-changing vehicles merges into. If lower, IntelliDrive again enhances flow. However, if the flow of the destined cluster is lower, the total flow increases only when the constructive effect of the reduced P-R time of drivers is able to compensate for the loss in flow due to the lane-changing maneuver, the case held in our example scenario above. Finally, even if the total flow was seen to reduce when using IntelliDrive, the total travel time of drivers is enhanced by letting them join higher-speed clusters.

\section{Conclusion}

In this paper, we investigated the effect of IntelliDrive on the efficiency of multi-lane highway systems. We studied how the gradual introduction of IntelliDrive-enabled vehicles can enhance the efficiency of traffic flow. Towards this goal, we developed a mobility model where vehicles moved in clusters inside which they constantly maintained a safety carfollowing distance with their leaders. Having justified the fact that IntelliDrive would reduce the perception-reaction time of drivers, traffic flow was seen to increase due to the lower lead spacing of the equipped vehicles. A tight lower bound for the flow of a cluster of a mixed variety of vehicles was also derived. This bound was shown to be independent of the number of vehicles inside the cluster. A weighted average of the per-cluster flows was then used as a metric to represent the flow over all clusters. Lane-changing mechanisms where incorporated into the model for multilane highways. In some cases, IntelliDrive could be utilized to help make lane changes which are otherwise not possible without such help. We argued that such assisted lane changes, although might not always increase the overall flow, would in any case decrease the travel time of the drivers. 


\section{Appendices}

\section{A. Derivation of the Gap-Acceptance Equation (3)}

Let vehicle $j$ be the leader vehicle in cluster $n+1$ which is therefore moving with the constant speed $V_{n+1}$. Then, its location evolves with time as $x_{j}(t)=x_{j}+V_{n+1} t$, where $x_{j}$ is vehicle $j$ 's location at the time vehicle $i$ wants to make a lanechange. Moreover, as vehicle $i$, after making the lane-change, accelerates with the rate $a_{i}$ to reach the speed $V_{n}$, its location evolves as $x_{i}(t)=0.5 a_{\mathrm{i}} t^{2}+V_{m} t+x_{i}$ for $t \in\left[0\left(V_{n}-V_{m}\right) / a_{i}\right]$, where $x_{i}$ is its initial location on the left lane (which is equal to the location of the last vehicle in cluster $n$ ). Note that here we have assumed that it takes vehicle $i$ zero time to carry out the lane-changing. To have no collision between cluster $n+1$ and vehicle $i$, we should have $x_{i}(t)-x_{j}(t) \geq L t \in$ [0 $\left.\left(V_{n}-V_{m}\right) / a_{i}\right]$. The latter is a degree 2 function which is positive before its first root. Hence, by letting the first root being greater than $\left(V_{n}-V_{m}\right) / a_{i}$ and some manipulations, (3) is obtained.

\section{B. Proof of Theorem 1}

Given that vehicles are assisted or automated with probability $\alpha$ and $\beta$, respectively, (corresponding to $100 \alpha \%$ and $100 \beta \%$ market penetration rates for the assisted and automated vehicles), the number of assisted and automated vehicles within cluster $i\left(N_{i}^{\alpha}\right.$ and $\left.N_{i}^{\beta}\right)$ would follow a binomial distribution, hence,

$$
\begin{aligned}
E\left[q_{i}\right]= & V_{i} \sum_{r=0}^{N_{i}} \sum_{s=0}^{N_{i}-r} E\left[\frac{N_{i}}{\sum_{j=1}^{N_{i}} S_{i j}} \mid N_{i}^{\beta}=r, N_{i}^{\alpha}=s\right] \\
& \times p\left(N_{i}^{\alpha}=s \mid N_{i}^{\beta}=r\right) p\left(N_{i}^{\beta}=r\right) \\
= & V_{i} N_{i} \sum_{r=0}^{N_{i}} \sum_{s=0}^{N_{i}-r} E\left[\frac{\left(\begin{array}{c}
N_{i}-r \\
s
\end{array}\right) \alpha^{s}(1-\alpha)^{N_{i}-r-s}}{\sum_{j=1}^{N_{i}} S_{i j}} \mid N_{i}^{\beta}=r, N_{i}^{\alpha}=s\right] \\
& \times p\left(N_{i}^{\beta}=r\right) \\
\stackrel{(1)}{\geq} & V_{i} N_{i} \sum_{r=0}^{N_{i}} \sum_{s=0}^{N_{i}-r} \frac{\left(\begin{array}{c}
N_{i}-r \\
s
\end{array}\right) \alpha^{s}(1-\alpha)^{N_{i}-r-s}}{E\left[\sum_{j=1}^{N_{i}} S_{i j} \mid N_{i}^{\beta}=r, N_{i}^{\alpha}=s\right]} p\left(N_{i}^{\beta}=r\right) \\
\stackrel{(2)}{=} & V_{i} N_{i} \sum_{r=0}^{N_{i}} \sum_{s=0}^{N_{i}-r} \frac{\left(\begin{array}{c}
N_{i}-r \\
s
\end{array}\right) \alpha^{s}(1-\alpha)^{N_{i}-r-s}}{a r+b \alpha s+c N_{i}} p\left(N_{i}^{\beta}=r\right) \\
= & V_{i} N_{i} \sum_{r=0}^{N_{i}} \frac{\left(\begin{array}{c}
N_{i} \\
r
\end{array}\right) \beta^{r}(1-\beta)^{N_{i}-r}}{\left(a-b \alpha^{2}\right) r+\left(c+b \alpha^{2}\right) n} \\
\stackrel{(3)}{\geq} & V_{i} N_{i} \sum_{r=0}^{N_{i}} \frac{p\left(N_{i}^{\beta}=r\right)}{a r+b \alpha\left(\alpha\left(N_{i}-r\right)\right)+c N_{i}}
\end{aligned}
$$

$$
\begin{aligned}
& \stackrel{(4)}{\geq} \frac{V_{i} N_{i}}{\left(a-b \alpha^{2}\right) \beta N_{i}+\left(c+b \alpha^{2}\right) N_{i}} \\
& =\frac{V_{i}}{c+a \beta+b \alpha^{2}(1-\beta)},
\end{aligned}
$$

where (2) is because according to the car-following spacing (7), we have

$$
\begin{aligned}
E\left[\sum_{j=1}^{N_{i}} S_{i j} \mid N_{i}^{\beta}=r, N_{i}^{\alpha}=s\right] \\
=r\left(G V_{i}^{2}+\mu_{\tau(\beta)} V_{i}+L\right)+s\left(G V_{i}^{2}+\mu_{\tau(\alpha)} V_{i}+L\right) \\
\quad+\left(N_{i}-r-s\right)\left(G V_{i}^{2}+\mu_{\tau(\alpha=0)} V_{i}+L\right) .
\end{aligned}
$$

which results in (2) when $a=V_{i}\left(\mu_{\tau(\beta)}-\mu_{\tau(\alpha=0)}\right), b=$ $V_{i}\left(\mu_{\tau(\alpha=1)}-\mu_{\tau(\alpha=0)}\right), c=G V_{i}^{2}+\mu_{\tau(\alpha=0)} V_{i}+L$. Note that all (1), (3) and (4) are due to Jensen's inequality. (Jensen's inequality states that $E[f(x)] \geq f(E[x])$ when $f$ is a convex function.) The use of Jensen's inequality in (3) is as follows:

$$
\begin{aligned}
& \sum_{s=0}^{N_{i}-r} \frac{\left(\begin{array}{c}
N_{i}-r \\
s
\end{array}\right) \alpha^{s}(1-\alpha)^{N_{i}-r-s}}{a r+b \alpha s+c N_{i}} \\
& \quad=E_{s}\left[\frac{1}{a r+b \alpha s+c N_{i}}\right] \\
& \quad \geq \frac{1}{a r+b \alpha E[s]+c N_{i}} \\
& \quad=\frac{1}{a r+b \alpha\left(\alpha\left(N_{i}-r\right)\right)+c N_{i}} .
\end{aligned}
$$

The expectation in the first equality is with respect to random variable $s$ which is drawn from a set of size $N_{i}-r$ according to a binomial distribution with parameter $\alpha$. This way the expected value of $s$ would be $\alpha\left(N_{i}-r\right)$ which is used to achieve the last equality. (4) is a result of applying the same ideas as above for the automated vehicles.

\section{Derivation of Equation (15)}

When $Y_{m n}=1$, according to definition, no vehicle from cluster $m$ can join cluster $n$ hence $\alpha_{m_{n+1}}^{r}=\alpha_{m_{n}}^{r}$. For the case where $Y_{m n=2}$, multiply both the numerator and the denominator by $N_{m_{n}}^{r}$, the number of vehicles in cluster $m$ at the time of encountering cluster $n$. Notice that here only the equipped vehicles could make the lane change. Thus, the numerator shows the expected number of assisted vehicles and the denominator the expected total number of vehicles that remain in cluster $m$ after being overtaken by cluster $n$. For the case where $Y_{m n}=3$, we define the following events. Let $A=\left\{V_{m}^{r} \leq v<V_{n}^{l}\right\}, B=\left\{V_{m}^{r} \leq v<V_{n_{m}^{\prime}}^{l}\right\}$, and 
$C=\left\{V_{m}^{r} \leq v<V_{n_{m}^{\prime \prime}}^{l}\right\}$. As $A \subseteq B \subseteq C$, we have $P(A \mid B)=$ $P(A) / P(B)$ and $P(A \mid C)=P(A) / P(C)$. Hence:

$$
\begin{aligned}
& \alpha_{m_{n+1}}^{r} \\
& =\frac{\alpha_{m_{n}}^{r} P(A \mid B) N_{m_{n}}^{r}}{\left(\alpha_{m_{n}}^{r}+\beta_{m_{n}}^{r}\right) P(A \mid B) N_{m_{n}}^{r}+\left(1-\alpha_{m_{n}}^{r}-\beta_{m_{n}}^{r}\right) P(A \mid C) N_{m_{n}}^{r}} \\
& =\frac{\alpha_{m_{n}}^{r} P(C)}{\left(\alpha_{m_{n}}^{r}+\beta_{m_{n}}^{r}\right) P(C-B)+P(B)}
\end{aligned}
$$

which yields the result.

\section{References}

[1] T. T. Institute, "2005 urban mobility report," 2005, http://. mobility.tamu.edu/ums/report/.

[2] BTS, "National transportation statistics 2006," 2006, http:// www.bts.gov/publications/national_transportation_statistics/ 2006/index.html.

[3] ITS, "Intellidrive," http://www.its.dot.gov/intellidrive/.

[4] D. Jiang, V. Taliwal, A. Meier, W. Holfelder, and R. Herrtwich, "Design of $5.9 \mathrm{GHz}$ DSRC-based vehicular safety communication," IEEE Wireless Communications, vol. 13, no. 5, pp. 36-43, 2006.

[5] Q. Xu, T. Mak, J. Ko, and R. Sengupta, "Vehicle-to-vehicle safety messaging in DSRC," in Proceedings of the 1st ACM International Workshop on Vehicular Ad Hoc Networks (VANET '04), pp. 19-28, Philadelphia, Pa, USA, October 2004.

[6] T. K. Mak, K. P. Laberteaux, and R. Sengupta, "A multichannel VANET providing concurrent safety and commercial services," in Proceedings of the 2nd ACM International Workshop on Vehicular Ad Hoc Networks (VANET '05), pp. 1-9, September 2005.

[7] M. Green, "How long does it take to stop? Methodological analysis of driver perception-brake times," Transportation Human Factors, vol. 2, no. 3, pp. 195-216, 2000.

[8] R. D. Luce, Response Times: Their Role in Inferring Elementary Mental Organization, Oxford University Press, New York, NY, USA, 1986.

[9] A. Vahidi and A. Eskandarian, "Research advances in intelligent collision avoidance and adaptive cruise control," IEEE Transactions on Intelligent Transportation Systems, vol. 4, no. 3, pp. 132-153, 2003.

[10] F. Michaud, P. Lepage, P. Frenette, D. Létourneau, and N. Gaubert, "Coordinated maneuvering of automated vehicles in platoons," IEEE Transactions on Intelligent Transportation Systems, vol. 7, no. 4, pp. 437-446, 2006.

[11] S. Darbha and K. R. Rajagopal, "Intelligent cruise control systems and traffic flow stability," Transportation Research Part C, vol. 7, no. 6, pp. 329-352, 1999.

[12] H.-S. Tan, R. Rajamani, and W.-B. Zhang, "Demonstration of an automated highway platoon system," in Proceedings of the American Control Conference, pp. 1823-1827, June 1998.

[13] D. Swaroop, J. Hedrick, C. C. Chien, and P. Ioannou, "Comparison of spacing and headway control laws for automatically controlled vehicles," Vehicle System Dynamics, vol. 23, no. 1, pp. 597-625, 1994.

[14] J. Carbaugh, D. N. Godbole, and R. Sengupta, "Safety and capacity analysis of automated and manual highway systems," Transportation Research Part C, vol. 6, no. 1-2, pp. 69-99, 1998.
[15] J. Zhou and H. Peng, "Range policy of adaptive cruise control vehicles for improved flow stability and string stability," IEEE Transactions on Intelligent Transportation Systems, vol. 6, no. 2, pp. 229-237, 2005.

[16] B. S. Y. Rao and P. Varaiya, "Flow benefits of autonomous intelligent cruise control in mixed manual and automated traffic," Transportation Research Board, vol. 1408, pp. 36-43, 1993.

[17] A. Touran, M. A. Brackstone, and M. McDonald, "A collision model for safety evaluation of autonomous intelligent cruise control," Accident Analysis and Prevention, vol. 31, no. 5, pp. 567-578, 1999.

[18] C. Tampere, S. Hoogendoorn, and B. van Arem, "A behavioural approach to instability, stop and go waves, wide jams and capacity drop," in Proceedings of the 16th International Symposium on Transportation and Traffic Theory.

[19] Q. Xu, T. Mak, J. Ko, and R. Sengupta, "Medium access control protocol design for vehicle-vehicle safety messages," IEEE Transactions on Vehicular Technology, vol. 56, no. 2, pp. 499$518,2007$.

[20] F. Li and Y. Wang, "Routing in vehicular ad hoc networks: a survey," IEEE Vehicular Technology Magazine, vol. 2, no. 2, pp. 12-22, 2007.

[21] M. Nekoui and H. Pishro-Nik, "Reliable inter-vehicle communications for vehicular ad hoc networks," in Proceedings of the 4th Annual International Wireless Internet Conference (WICON '08), 2008.

[22] D. Helbing, A. Hennecke, V. Shvetsov, and M. Treiber, "Microand macro-simulation of freeway traffic," Mathematical and Computer Modelling, vol. 35, no. 5-6, pp. 517-547, 2002.

[23] S. P. Hoogendoorn and P. H. L. Bovy, "Continuum modeling of multiclass traffic flow," Transportation Research Part B, vol. 34, no. 2, pp. 123-146, 2000.

[24] W. L. Jin, Kinematic wave models of network vehicular traffic, Ph.D. thesis, University of California, Davis, Calif, USA, 2003, http://arxiv.org/abs/math.DS/0309060.

[25] L. A. Pipes, "Car following models and the fundamental diagram of road traffic," Transportation Research, vol. 1, no. 1, pp. 21-29, 1967.

[26] R. Chandler, R. Herman, and E. Montroll, "Traffic dynamics: studies in car following," Operations Research, vol. 6, no. 2, pp. 165-184, 1958.

[27] D. C. Gazis, R. Herman, and R. W. Rothery, "Non-linear follow the leader models of traffic flow," Operations Research, vol. 9, pp. 545-567, 1961.

[28] P. G. Gipps, "A behavioural car-following model for computer simulation," Transportation Research Part B, vol. 15, no. 2, pp. 105-111, 1981.

[29] A. D. May, "Traffic flow fundamentals," in Microscopic Density Characteristics, pp. 162-177, Prentice Hall, 1990. 

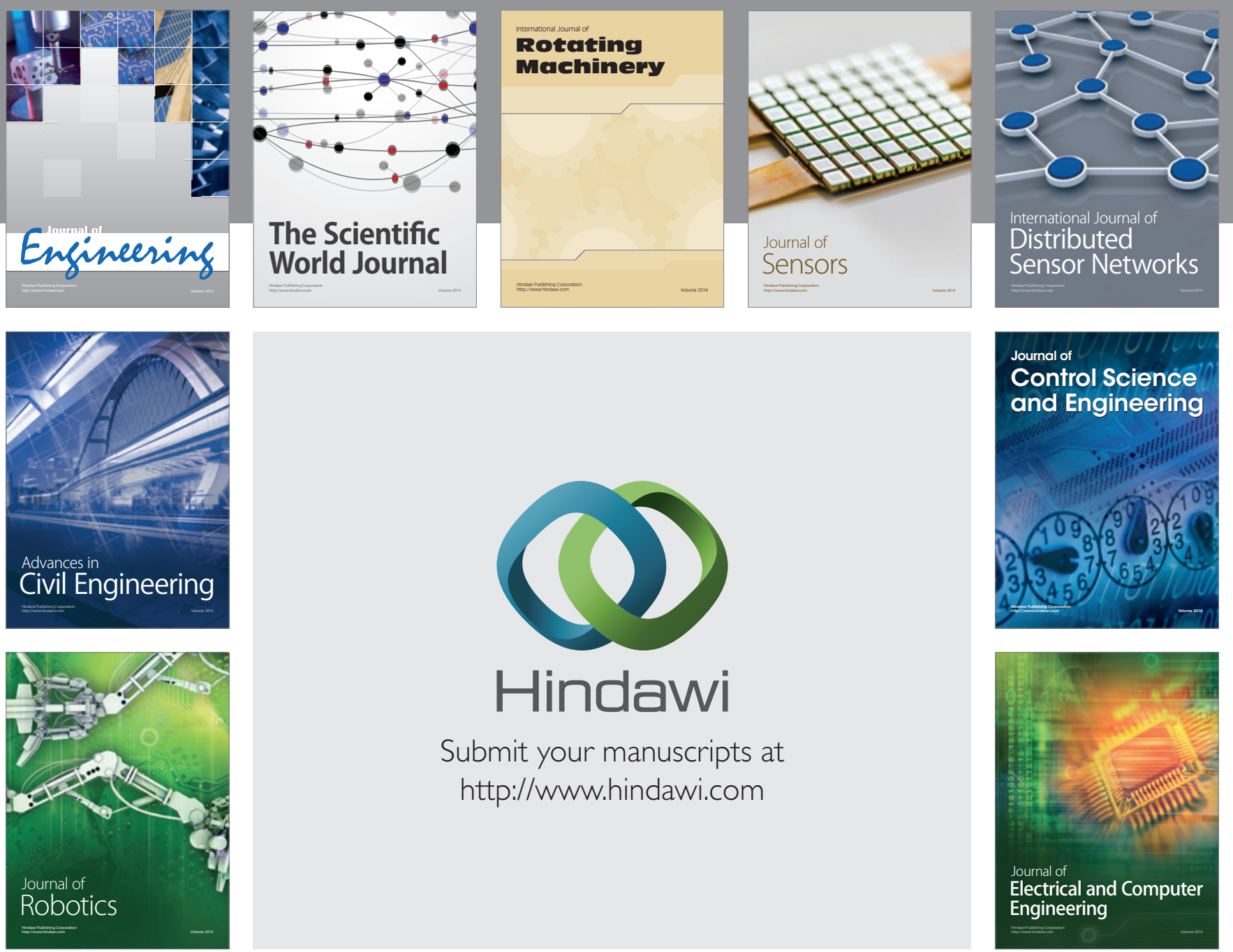

Submit your manuscripts at

http://www.hindawi.com
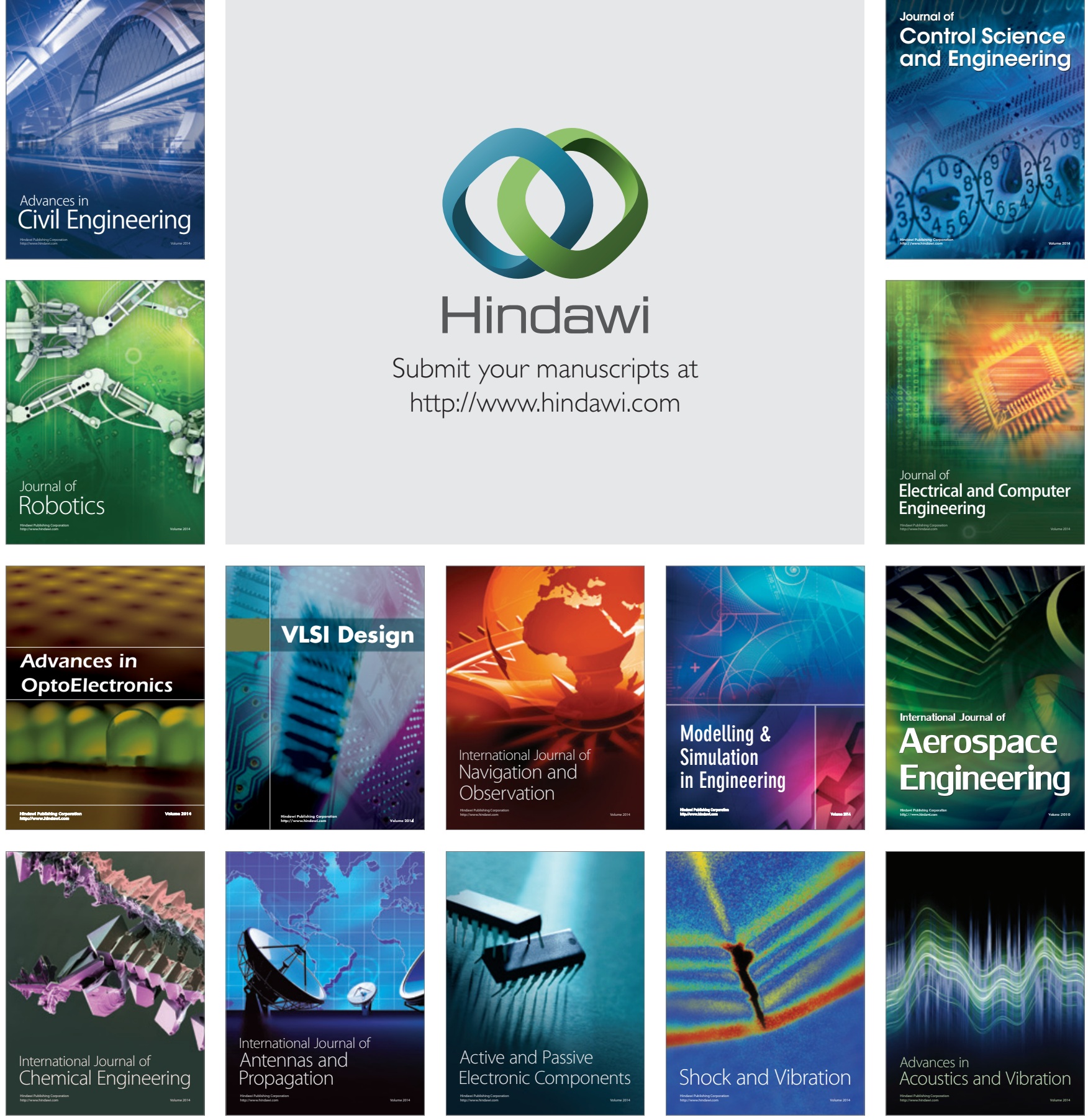